\title{
Effect of single nucleotide polymorphism on the total number of piglets born per parity of three different pig breeds
}

\author{
Kyoung-Tag Do ${ }^{1, a}$, Soon-Woo Jung ${ }^{2, a}$, Kyung-Do Park ${ }^{3, *}$, and Chong-Sam Na ${ }^{3, *}$
}

\author{
* Corresponding Authors: \\ Kyung-Do Park \\ Tel: +82-63-270-5934, Fax: +82-63-270-5936, \\ E-mail: doobalo@jbnu.ac.kr \\ Chong-Sam Na \\ Tel: +82-63-270-2607, Fax: +82-63-270-2614 \\ E-mail: csna@jbnu.ac.kr \\ 1 Department of Animal Biotechnology, Jeju National \\ University, Jeju 63243, Korea \\ 2 Hamyang-guncheong, Hamyang 50031, Korea \\ ${ }^{3}$ Department of Animal Biotechnology, Chonbuk \\ National University, Jeonju 54896, Korea \\ a These authors contributed equally to this work. \\ ORCID \\ Kyung-Do Park \\ https://orcid.org/0000-0002-1945-6708 \\ Chong-Sam Na \\ https://orcid.org/0000-0002-8979-5633
}

Submitted Jan 12, 2017; Revised Mar 29, 2017; Accepted Jul 28, 2017
Objective: To determine the effects of genomic breeding values (GBV) and single nucleotide polymorphisms (SNP) on the total number of piglets born (TNB) in 3 pig breeds (Berkshire, Landrace, and Yorkshire).

Methods: After collecting genomic information (Porcine SNP BeadChip) and phenotypic TNB records for each breed, the effects of GBV and SNP were estimated by using single step best linear unbiased prediction (ssBLUP) method.

Results: The heritability estimates for TNB in Berkshire, Landrace, and Yorkshire breeds were $0.078,0.107$, and 0.121 , respectively. The breeding value estimates for TNB in Berkshire, Landrace, and Yorkshire breeds were in the range of -1.34 to 1.47 heads, -1.79 to 1.87 heads, and -2.60 to 2.94 heads, respectively. Of sows having records for TNB, the reliability of breeding value for individuals with SNP information was higher than that for individuals without SNP information. Distributions of the SNP effects on TNB did not follow gamma distribution. Most SNP effects were near zero. Only a few SNPs had large effects. The numbers of SNPs with absolute value of more than 4 standard deviations in Berkshire, Landrace, and Yorkshire breeds were 11, 8 , and 19, respectively. There was no SNP with absolute value of more than 5 standard deviations in Berkshire or Landrace. However, in Yorkshire, four SNPs (ASGA 0089457, ASGA0103374, ALGA0111816, and ALGA0098882) had absolute values of more than 5 standard deviations.

Conclusion: There was no common SNP with large effect among breeds. This might be due to the large genetic composition differences and the small size of reference population. For the precise evaluation of genetic performance of individuals using a genomic selection method, it may be necessary to establish the appropriate size of reference population.

Keywords: Berkshire; Genomic Breeding Value; Landrace; Single Nucleotide Polymorphism; Total Number of Piglets Born; Yorkshire

\section{INTRODUCTION}

Since the deoxyribonucleic acid (DNA) structure was identified in 1950s, exploration technology for genetic variation of organisms has been developed rapidly due to rapid development of molecular biology technology with many genome projects to identify genome-wide base pair sequence. Due to the development of DNA chip technology using microarray which enables exhaustive analysis of several hundreds to millions of single nucleotide polymorphism (SNP) markers through selective hybridization on solid surface based on by genotype, many genes can be identified in a short period. Currently, the imputation of genotypes using higher density chips from low-density chips is being undertaken. Meuwissen et al [1] and Van Eenennaam et al [2] have proposed the genomic selection method using genome-wide high-density SNP markers for the first time.

Genetic performance of individuals can be predicted by genomic selection through marker 
mapping with dense interval. This is more accurate than conventional breeding value estimation method. Especially, it is highly accurate for breeding value estimation of young animals without phenotypic data, thus enabling juvenile selection [1]. Gengler et al [3] have proposed an algorithm to predict genomic information of animals without phenotypic data. VanRaden [4] has suggested an algorithm to calculate genomic relationship coefficient matrix and estimate genomic breeding value (GBV). Also, Misztal et al [5] have reported an algorithm that combines the conventional pedigree information with genomic information. Recently, Liu et al [6] have developed an SNP Single-step genomic model as a method to estimate SNP effects directly from the analysis model.

In this experiment, genomic information and phenotypic data on the total number of piglets born were collected from Berkshire, Landrace, and Yorkshire breeds. Their GBVs were estimated and the accuracies of these estimated breeding values were compared. In addition, SNP effects on total number of piglets born (TNB) by pig breed were investigated.

\section{MATERIALS AND METHODS}

\section{SNP data and quality control}

Using porcine SNP60 (v1, v2) manufactured by Illumina company and genomic profiler for porcine high density (GGP Porcine HD) genotyping BeadChip manufactured by GeneSeek company, genomic information for 3,998 breeding pigs was collected for Berkshire (1,903 heads), Landrace (1,041 heads), and Yorkshire (1,054 heads) breeds.

For quality control, SNPs on sex chromosome, SNPs without location information on chromosome, markers with more than $10 \%$ of missing rate, markers without polymorphism (homo or hetero genotype markers), markers with less than $1 \%$ of minor allele frequency, and markers with more than $23.93\left(\mathrm{p}<10^{-6}\right)$ of Hardy-Weinberg disequilibrium chi-square value, and genomic information of animals with more than $10 \%$ of SNP missing rate were excluded.

After performing quality control, the number of effective SNPs used for the analysis was 31,354 for Berkshire, 36,392 for Landrace, and 40,783 for Yorkshire. The numbers of pigs with genomic information for Berkshire, Landrace, and Yorkshire breeds were $1,871,1,038$, and 1,035 heads, respectively. Of sows with genomic information, the numbers of pigs with phenotypic data for Berkshire, Landrace, and Yorkshire breeds were 546,836 , and 898 heads, respectively (Table 1). Boars and candidate pigs only had SNP information. Phenotypic data were unavailable.

\section{Phenotypic data}

A total of 17,007 records of phenotypic data for the TNB were collected from Berkshire (4,504 records from 1,106 heads), Landrace (5,178 records from 1,498 heads), and Yorkshire
Table 1. Description of single nucleotide polymorphism (SNP) dataset

\begin{tabular}{lccc}
\hline \multirow{2}{*}{ Description } & \multicolumn{3}{c}{ Breed } \\
\cline { 2 - 4 } & 1,903 & 1,041 & 1,054 \\
\hline Total No. of animals & 32 & 3 & 19 \\
No. of animals with missing over $10 \%$ & 1,871 & 1,038 & 1,035 \\
No. of selected animals & 546 & 836 & 898 \\
No. of sows with record & 42,276 & 48,245 & 51,984 \\
No. of common markers on autosome & 31,354 & 36,392 & 40,783 \\
No. of selected (useful) markers & & & \\
\hline
\end{tabular}

(7,325 records from 1,923 heads) breeds. The total number of pigs was 3,600 Berkshires, 1,952 Landraces, and 2,424 Yorkshires. The average TNB for Berkshire, Landrace, and Yorkshire breeds were $8.58,11.92$, and 12.66 heads, respectively.

\section{Statistical model}

Estimation of genomic breeding values: For fixed effect, parity and farrowing year-month-week were included and the following analysis model was used:

$$
y=X b+Z a+W p+e
$$

Where, $y=\mathrm{n} \times 1$ vector of observation, $b=p \times 1$ vector of fixed effect, $a=q \times 1$ vector of additive genetic random effect, $p=q \times 1$ vector of permanent environmental random effect, $e$ $=n \times 1$ vector of residual effect, $\mathrm{X}(\mathrm{n} \times \mathrm{p}), \mathrm{Z}(\mathrm{n} \times \mathrm{q})$, and $\mathrm{W}(\mathrm{n} \times \mathrm{q})$ were known incidence matrix corresponding to $b, a$, and $p$, respectively.

Mixed model equation was as follows:

$$
\left[\begin{array}{ccc}
X^{\prime} X & X^{\prime} Z & X^{\prime} W \\
Z^{\prime} X & Z^{\prime} Z+\alpha_{1} H^{-1} & Z^{\prime} W \\
W^{\prime} X & W^{\prime} Z & W^{\prime} W+\alpha_{2} I
\end{array}\right]\left|\begin{array}{c}
\hat{b} \\
\hat{a} \\
\hat{p}
\end{array}\right|=\left[\begin{array}{c}
X^{\prime} y \\
Z^{\prime} y \\
W^{\prime} y
\end{array}\right]
$$

Where, $\alpha_{1}=\sigma_{e}^{2} / \sigma_{a}^{2}, \alpha_{2}=\sigma_{e}^{2} / \sigma_{p e}^{2}, \mathrm{H}^{-1}=\mathrm{A}^{-1}+\left[\begin{array}{cc}0 & 0 \\ 0 & G^{-1}-A_{22}^{-1}\end{array}\right]$, $\mathrm{A}^{-1}=$ inverse matrix of numerator relationship matrix, $\mathrm{G}^{-1}=$ inverse matrix of genomic relationship matrix and $A_{22}^{-1}=$ inverse matrix of numerator relationship matrix of pigs with genomic information. Reliability $\left(r^{2}\right)$ of breeding value was calculated using the prediction error variance (PEV) value. $r^{2}=1-\left(P E V / \sigma_{a}^{2}\right)$.

SNP effect: The SNP effect of each marker was estimated through reverse operation method of GBVs. When $\widehat{a_{g}}=Z \hat{u}$, $\widehat{a_{g}}=\mathrm{GBV}$ vector, $\mathrm{Z}=$ coefficient matrix of SNP, $\widehat{u}=$ vector of SNP effects.

If this equation is converted into variance equation, then $\mathrm{G}^{*}=\mathrm{ZDZ} \lambda$, where $\mathrm{D}$ was weighted vector. Using the above two equations, it can be converted into $\widehat{u}=\lambda D Z^{\prime} G^{*-1} \widehat{a_{g}}=$ $D Z^{\prime}(Z D Z)^{-1} \widehat{a_{g}}$, where $\widehat{u}=$ vector of SNP effects, $\widehat{a_{g}}=$ vector of GBV, $Z$ = coefficient matrix of SNP, $\mathrm{D}=$ weighted vector 
$[7,8]$.

\section{RESULTS AND DISCUSSION}

\section{Genetic parameter}

The heritability estimates for TNB of Berkshire, Landrace, and Yorkshire breeds were $0.078,0.107$, and 0.121 , respectively. The repeatability estimates for TNB of Berkshire, Landrace, and Yorkshire breeds were $0.176,0.190$, and 0.188 , respectively (Table 2). Su et al [9] and Chen et al [10] have reported that the heritability estimates for TNB of Landrace breed is 0.08 . The heritability estimates for TNB of Yorkshire and Duroc breeds have been reported to be 0.10 and 0.09 , respectively [10]. It is 0.07 in Berkshire breed [11]. Therefore, the heritability for TNB seems to be low or around 0.1, depending on genetic characteristics of the population [12].

\section{Breeding value and reliability}

The breeding value estimates for TNB of Berkshire, Landrace, and Yorkshire breeds were in the range of -1.34 to $1.47,-1.79$ to 1.87 , and -2.60 to 2.94 heads, respectively. The reliabilities for the estimated breeding values depended on the number of records, pedigree information, and heritability. Reliability for the breeding value was estimated after classifying sows into two groups (with or without SNP information). Of sows with records for the TNB, the reliability of the breeding value for individuals with SNP information was higher than that for individuals without SNP information (Table 3).

This result was in agreement with that of Forni et al [13] showing that higher reliability is obtained for individuals with SNP information. When genomic information is used, reliability is increased. Especially, when breeding values for the progeny without phenotypic data are estimated using conventional BLUP method, their breeding values are equal to the average of breeding values of their parents. Breeding values for all progenies from the same parents are the same. On the other hand, for the estimation of GBV, genomic information of individuals considering Mendelian sampling is used. Therefore, more precise breeding value can be obtained. This seems to be very efficient for the selection of candidate pigs [14].

\section{SNP effects}

In ssBLUP, both phenotypic data of individuals with genomic
Table 2. Additive genetic $\left(\sigma_{\mathrm{a}}^{2}\right)$, permanent environmental $\left(\sigma_{\text {pe }}^{2}\right)$, residual $\left(\sigma_{\mathrm{e}}^{2}\right)$ variance components, heritabilities $\left(h^{2}\right)$, standard error $(\mathrm{SE})$, and repeatabilities $(r)$ for the total number of piglets born (TNB) by breed

\begin{tabular}{|c|c|c|c|c|c|}
\hline \multirow{2}{*}{ Breed } & \multicolumn{3}{|c|}{ Variance component } & \multirow{2}{*}{$\mathrm{h}^{2} \pm \mathrm{SE}$} & \multirow[b]{2}{*}{$r$} \\
\hline & $\sigma_{a}^{2}$ & $\sigma_{\mathrm{pe}}^{2}$ & $\sigma_{\mathrm{e}}^{2}$ & & \\
\hline Berkshire & 0.6024 & 0.7516 & 6.3597 & $0.078 \pm 0.021$ & 0.176 \\
\hline Landrace & 0.9861 & 0.7641 & 7.4400 & $0.107 \pm 0.024$ & 0.190 \\
\hline Yorkshire & 1.4545 & 0.8143 & 9.7902 & $0.121 \pm 0.021$ & 0.188 \\
\hline
\end{tabular}

information and phenotypic data of individuals with only pedigree information can be used $[15,16]$. For ssBLUP, since the estimated effects from the model are the pedigree and GBVs of the individuals, conventional breeding value and GBV can be estimated simultaneously by one analysis. Through back solution, the effects of SNPs can be estimated reversely $[7,8]$.

After absolute values for the estimated SNP effects were taken, optimal histograms fitted with real estimated values were drawn using the estimated parameters for gamma distribution (Figure 1). They were distributions for SNP effects on TNB. Most SNP effects were near zero. Only a few SNPs had large effects.

Meuwissen et al [1] have reported that the distribution of quantitative trait locus follows Gamma distribution. However, in this study, we performed Goodness-of-Fit tests for gamma distribution and found that the distribution of estimated SNP effects on TNB did not follow Gamma distribution (Table 4).

\section{Genome-wide association study}

To compare the relative magnitude of estimated SNP effects, absolute values of estimated SNP effects were taken after standardization (Figure 2). If we assume that $99.74 \%$ of values drawn from a normal distribution are within 3 standard deviations, selection threshold should be more than 4 standard deviations as useful markers (Table 5). For TNB, the numbers of SNPs with absolute value of more than 4 standard deviations in Berkshire, Landrace, and Yorkshire breeds were 11, 8, and 19, respectively. There was no SNP with absolute value of more than 5 standard deviations in Berkshire or Landrace breed. However, in Yorkshire, four SNPs (ASGA0089457 [5,20], ASGA0103374 [5.11], ALGA0111816 [5.32], and ALGA 0098882 [5.79]) had absolute values of more than 5 standard deviations (Table 5). No SNP had large effects for all breeds.

Table 3. Reliabilities $\left(r_{2}\right)$ on the estimated breeding values of animals with SNPs or without SNPs in sows with total number of piglets born (TNB) records by breed

\begin{tabular}{|c|c|c|c|c|c|c|}
\hline \multirow{2}{*}{ Breed } & \multicolumn{2}{|c|}{ Overall } & \multicolumn{2}{|c|}{ Without SNPs } & \multicolumn{2}{|c|}{ with SNPs } \\
\hline & Animals & $r^{2} \pm S D$ & Animals & $r^{2} \pm S D$ & Animals & $r^{2} \pm S D$ \\
\hline Berkshire & 1,106 & $0.33 \pm 0.08$ & 564 & $0.30 \pm 0.08$ & 542 & $0.35 \pm 0.08$ \\
\hline Landrace & 1,498 & $0.41 \pm 0.10$ & 662 & $0.33 \pm 0.08$ & 836 & $0.48 \pm 0.07$ \\
\hline Yorkshire & 1,923 & $0.45 \pm 0.11$ & 1,025 & $0.39 \pm 0.10$ & 898 & $0.53 \pm 0.07$ \\
\hline
\end{tabular}

SNPs, single nucleotide polymorphisms; SD, standard deviation. 

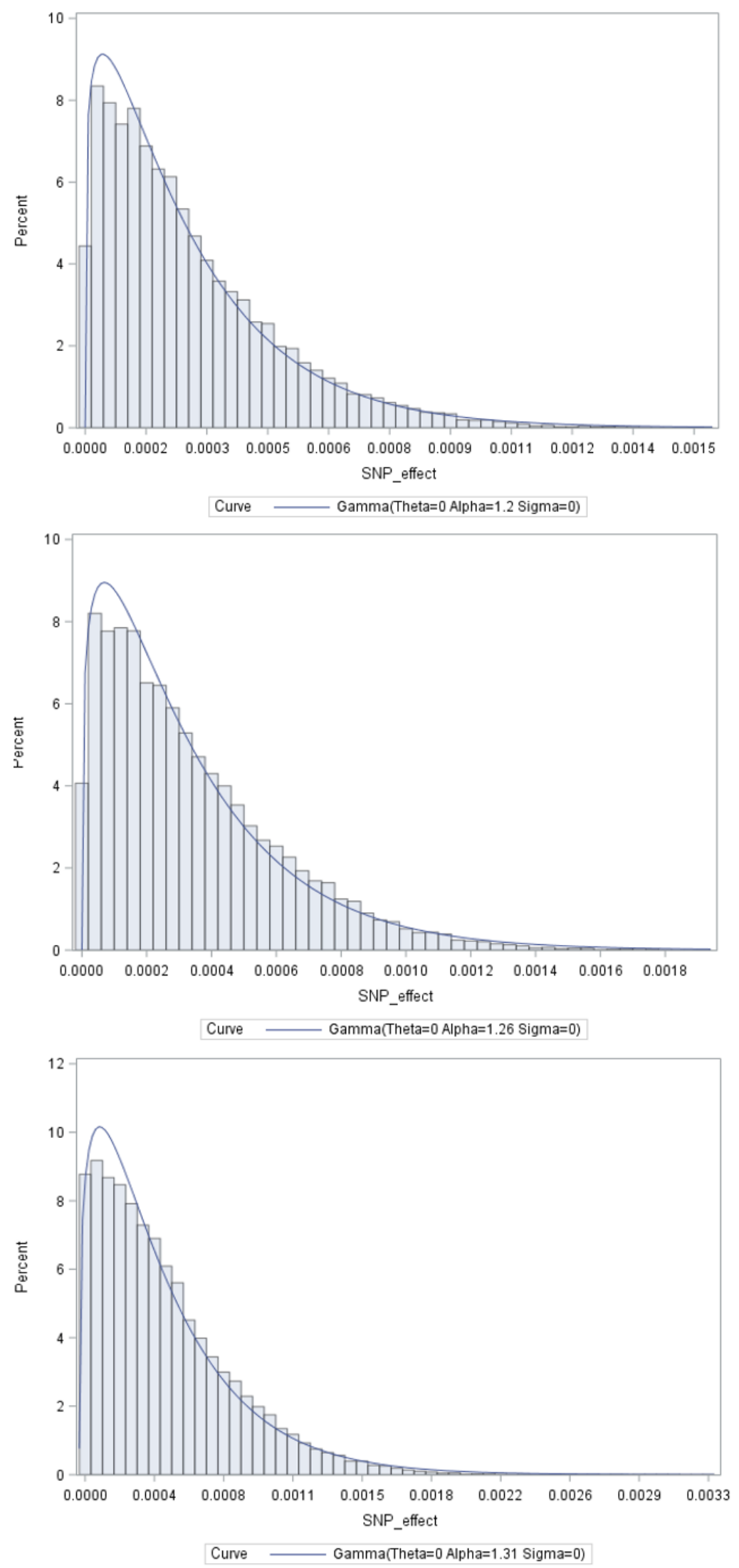

Figure 1. Distribution of estimated single nucleotide polymorphism (SNP) effects for the total number of piglets born (TNB) in Berkshire, Landrace, and Yorkshire breeds (from left to right). 
Table 4. Goodness-of-fit tests for gamma distribution of estimated single nucleotide polymorphism (SNP) effects for the total number of piglets born (TNB) by breed

\begin{tabular}{lccc}
\hline \multirow{2}{*}{ Breed } & \multicolumn{3}{c}{ Test method (statistics) } \\
\cline { 2 - 4 } & $\begin{array}{c}\text { Kolmogorov-Smirnov Cramer-von Mises } \\
\text { (D) }\end{array}$ & $\begin{array}{c}\text { Anderson-Darling } \\
\text { (W-Sq) }\end{array}$ \\
\hline Berkshire & $0.0241^{* *}$ & $5.7569^{* *}$ & $33.9518^{* *}$ \\
Landrace & $0.0240^{* *}$ & $8.1674^{* *}$ & $49.8215^{* *}$ \\
Yorkshire & $0.0285^{* *}$ & $10.5087^{* *}$ & $60.4243^{* *}$ \\
\hline
\end{tabular}

** $p<0.01$.

This might be due to the large genetic composition differences among breeds.
Only a few markers had large effects on the TNB. Due to linkage disequilibrium, some markers in regions adjacent to markers with large effects also had large effects. For TNB, SNPs in chromosome number 2 (MARC0046316, ASGA0101159), chromosome number 6 (ALGA0114670, H3GA0055046), and chromosome number 17 (ALGA0093629, ASGA0075678, ALGA0093681) in Berkshire, SNPs in chromosome number 7 (ASGA0036842, ALGA0045470) in Landrace, and SNPs in chromosome number 3 (MARC0053067, MARC0034058, ASGA0089809, H3GA0009642), chromosome number 8 (ASGA0089457, ASGA0103374, ALGA0111816, H3GA00 25815), and chromosome number 18 (M1GA0023425, ALGA 0098863, H3GA0051231, ALGA0109739, ALGA0098882, ALGA

Table 5. Single nucleotide polymorphism (SNP) name, chromosome number (Chr.), position, SNP effect and absolute standardized SNP effect of more than 4.0 STD value for the total number of piglets born by breed

\begin{tabular}{|c|c|c|c|c|c|}
\hline Breed & SNP name & Chr. & Position & SNP effect & |STD value| \\
\hline \multirow[t]{11}{*}{ Berkshire } & MARC0046316 & 2 & 2216738 & 0.00140875 & 4.39 \\
\hline & ASGA0101159 & 2 & 2224107 & 0.00135236 & 4.22 \\
\hline & H3GA0056247 & 3 & 101704758 & -0.00149613 & 4.40 \\
\hline & ALGA0114670 & 6 & 74627362 & -0.00138505 & 4.06 \\
\hline & H3GA0055046 & 6 & 74682817 & -0.00138505 & 4.06 \\
\hline & DRGA0012350 & 13 & 53111671 & 0.00128118 & 4.01 \\
\hline & ASGA0096197 & 16 & 77537650 & 0.00130050 & 4.06 \\
\hline & ALGA0105626 & 17 & 18894036 & 0.00145942 & 4.54 \\
\hline & ALGA0093629 & 17 & 21259716 & -0.00145520 & 4.28 \\
\hline & ASGA0075678 & 17 & 21701959 & 0.00129377 & 4.04 \\
\hline & ALGA0093681 & 17 & 22559386 & -0.00154334 & 4.54 \\
\hline \multirow[t]{8}{*}{ Landrace } & H3GA0016445 & 5 & 45404530 & -0.00193198 & 4.37 \\
\hline & ASGA0036842 & 7 & 126525777 & 0.00171787 & 4.01 \\
\hline & ALGA0045470 & 7 & 126664035 & 0.00173147 & 4.04 \\
\hline & MARC0043234 & 13 & 119326691 & -0.00179717 & 4.06 \\
\hline & H3GA0038201 & 13 & 211949439 & 0.00175716 & 4.10 \\
\hline & DIAS0003382 & 15 & 83795094 & -0.00179399 & 4.05 \\
\hline & ALGA0103750 & 15 & 142396418 & 0.00177819 & 4.14 \\
\hline & ASGA0075659 & 17 & 21411732 & -0.00177623 & 4.01 \\
\hline \multirow[t]{19}{*}{ Yorkshire } & M1GA0002671 & 2 & 10286572 & 0.00228474 & 4.10 \\
\hline & MARC0053067 & 3 & 56950047 & -0.00280437 & 4.93 \\
\hline & MARC0034058 & 3 & 57211150 & -0.00240863 & 4.23 \\
\hline & ASGA0089809 & 3 & 58664523 & -0.00243213 & 4.27 \\
\hline & H3GA0009642 & 3 & 58873656 & -0.00277533 & 4.88 \\
\hline & ASGA0089457 & 8 & 146074727 & -0.00295488 & 5.20 \\
\hline & ASGA0103374 & 8 & 146101319 & -0.00290477 & 5.11 \\
\hline & ALGA0111816 & 8 & 146216038 & -0.00302276 & 5.32 \\
\hline & H3GA0025815 & 8 & 146688242 & -0.00233696 & 4.11 \\
\hline & CASI0008334 & 9 & 116927941 & 0.00227066 & 4.07 \\
\hline & DRGA0010231 & 10 & 9488500 & -0.00246896 & 4.34 \\
\hline & H3GA0038333 & 14 & 2865914 & -0.00251473 & 4.42 \\
\hline & ALGA0098185 & 18 & 45821014 & 0.00233614 & 4.19 \\
\hline & M1GA0023425 & 18 & 57244090 & -0.00233631 & 4.10 \\
\hline & ALGA0098863 & 18 & 57260307 & -0.00250486 & 4.40 \\
\hline & H3GA0051231 & 18 & 57292109 & -0.00250486 & 4.40 \\
\hline & ALGA0109739 & 18 & 57411145 & 0.00252987 & 4.53 \\
\hline & ALGA0098882 & 18 & 57936786 & -0.00328544 & 5.79 \\
\hline & ALGA0098883 & 18 & 57957917 & -0.00266136 & 4.68 \\
\hline
\end{tabular}



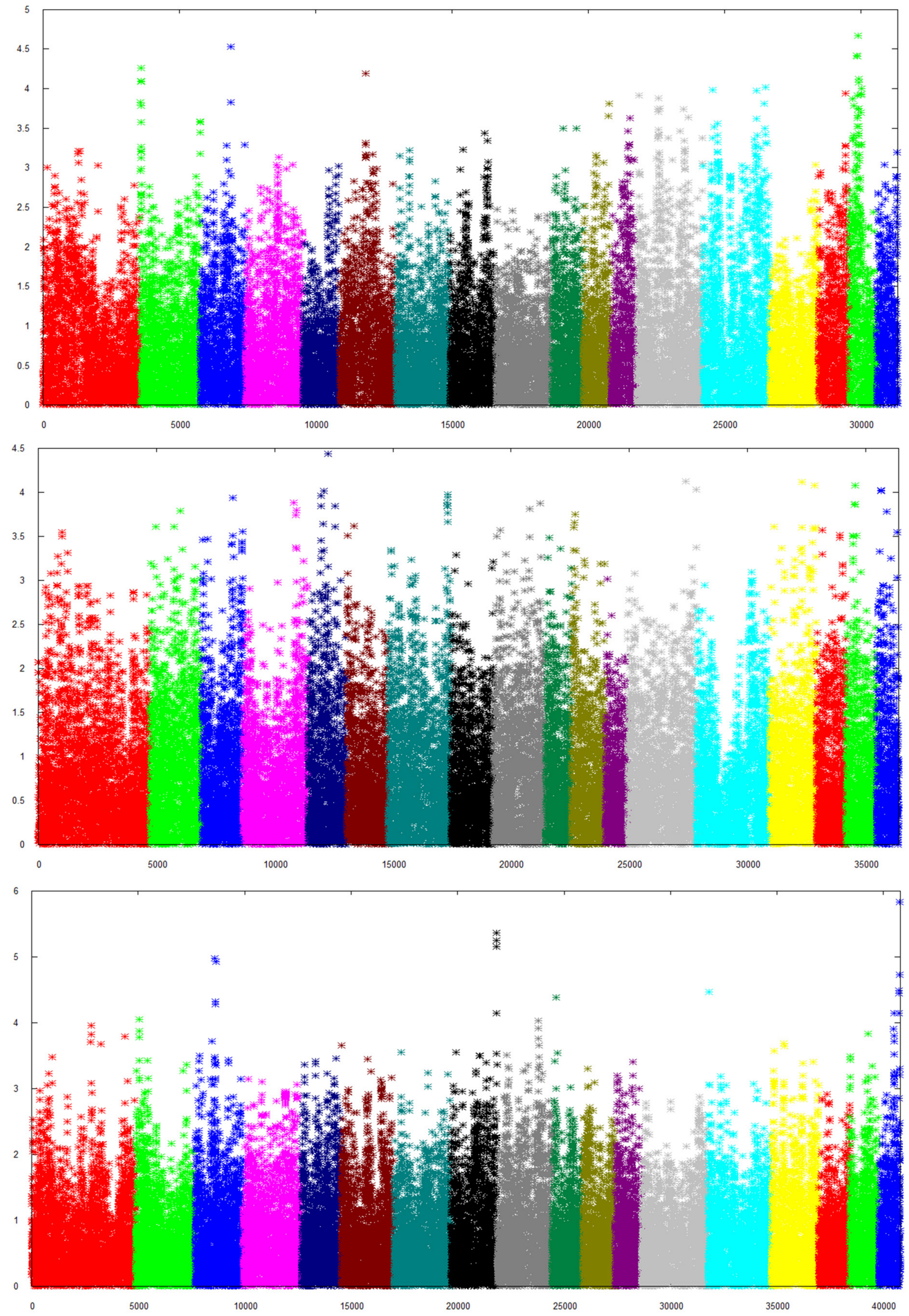

Figure 2. Manhattan plots for standardized single nucleotide polymorphism (SNP) effects on the total number of piglets born (TNB) in Berkshire, Landrace, and Yorkshire breeds (from upper to lower). 
0098883) in Yorkshire were in linkage disequilibrium blocks. Guo et al [17] reported that in Landrace, 18 SNPs on chromosome 2, 5 SNPs on chromosome 3, 7 SNPs on chromosome 6, 1 SNP on chromosome 13, and 3 SNPs on chromosome 14 had effects on the TNB, while in Yorkshire, 9 SNPs on chromosome 1 and 2 SNPs on chromosome 3 had effects on the TNB. Also, it was reported that in Landrace, 5 SNPs on chromosome 9 had effects on the total number of piglets born [18]. However, SNPs which were identified to have effects on the TNB in this experiment were not reported in other experiments.

It has been known that in Berkshire, complement C1q B chain gene (ALGA0114670) with high SNP effect is the candidate gene which is associated with the immune responses to porcine reproductive and respiratory syndrome virus infection and affects reproductive immunity in pigs [19,20], and in Landrace, phospholipase D1 gene (MARC0043234) controls the $\mathrm{mTORC} 1$ regulators which play a crucial role for the regulation of skeletal muscle protein synthesis in neonatal pigs [21]. Also, it was reported that in Yorkshire, CD6 molecule gene (M1GA0002671) regulates the cell adhesion molecules expression in the biological pathways including the development of embryonic cells and nerve tissues, and protein kinase, cGMP-dependent, type II (ASGA0089457, ASGA0103374) is involved in the secretion of luteinizing hormone beta polypeptide and progesterone [22].

There was no common SNP with large effect among breeds. This might be due to the large genetic composition differences among breeds. If we analyze again after deleting markers in the linkage disequilibrium blocks, the effect will be clearer. The main reason for that may be the small size of reference population used in this study, which could happen in any study when the size of reference population is small. For the precise evaluation of genetic performance of individuals using a genomic selection method, it may be necessary to establish the appropriate size of reference population.

\section{CONFLICT OF INTEREST}

We certify that there is no conflict of interest with any financial organization regarding the material discussed in the manuscript.

\section{ACKNOWLEDGMENTS}

This work was supported by a grant (PJ01322201) of the Next Generation BioGreen 21 Program funded by Rural Development Administration, Republic of Korea.

\section{REFERENCES}

1. Meuwissen THE, Hayes BJ, Goddard ME. Prediction of total genetic value using genome-wide dense marker maps. Genetics 2001;157:1819-29.

2. Van Eenennaam AL, Weigel KA, Young AE, Cleveland MA, Dekkers JCM. Applied animal genomics: results from the field. Anim Biosci 2014;2:105-39.

3. Gengler N, Abras S, Verkenne C, et al. Accuracy of prediction of gene content in large animal populations and its use for candidate gene detection and genetic evaluation. J Dairy Sci 2008;91:1652-9.

4. VanRaden PM. Efficient methods to compute genomic predictions. J Dairy Sci 2008;91:4414-23.

5. Misztal I, Legarra A, Aguilar I. A relationship matrix including full pedigree and genomic information. J Dairy Sci 2009;92: 4656-3.

6. Liu M, Goddard ME, Reinhardt F, Reent R. A single-step genomic model with direct estimation of marker effects. J Dairy Sci 2014;97:5833-50.

7. Christensen OF, Madsen P, Nielsen B, Ostersen T, Su G. Singlestep methods for genomic evaluation in pigs. Animal 2012;6: 1565-71.

8. Wang H, Misztal I, Aguilar I, et al. Genome-wide association mapping including phenotypes from relatives without genotypes in a single-step (ssGWAS) for 6-week body weight in broiler chickens. Front Genet 2014;5:134.

9. Su G, Lund MS, Sorensen D. Selection for litter size at day five to improve litter size at weaning and piglet survival rate. J Anim Sci 2007;85:1385-92.

10. Chen P, Baas TJ, Mabry JW, Koehler KJ, Dekkers JC. Genetic parameters and trends for litter traits in U.S. Yorkshire, Duroc, Hampshire, and Landrace pigs. J Anim Sci 2003;81:46-53.

11. Lee JH, Song KD, Lee HK, et al. Genetic parameters of reproductive and meat quality traits in Korean Berkshire pigs. AsianAustralas J Anim Sci 2015;28:1388-93.

12. Arango J, Misztal I, Tsuruta S, Culbertson M, Herring W. Threshold-linear estimation of genetic parameters for farrowing mortality, litter size, and test performance of Large White sows. J Anim Sci 2005;83:499-506.

13. Forni S, Aguilar I, Misztal I. Different genomic relationship matrices for single-step analysis using phenotypic, pedigree and genomic information. Genet Sel Evol 2011;43:1.

14. Clark SA, Kinghorn BP, Hickey JM, van der Werf JH. The effect of genomic information on optimal contribution selection in livestock breeding programs. Genet Sel Evol 2013;45:1.

15. Legarra A, Aguilar I, Misztal I. A relationship matrix including full pedigree and genomic information. J Dairy Sci 2009;92: 4656-63.

16. Aguilar I, Misztal I, Johnson DL, et al. A unified approach to utilize phenotypic, full pedigree, and genomic information for genetic evaluation of Holstein final score. J Dairy Sci 2010;93: 743-52.

17. Guo X, Su G, Christensen OF, Janss L, Lund MS. Genome-wide association analyses using a Bayesian approach for litter size 
and piglet mortality in Danish Landrace and Yorkshire pigs. BMC Genomics 2016;17:468.

18. Uimari P, Sironen A, Sevón-Aimonen ML. Whole-genome SNP association analysis of reproduction traits in the Finnish Landrace pig breed. Genet Sel Evol 2011;43:42.

19. Wysocki M, Chen H, Steibel JP, et al. Identifying putative candidate genes and pathways involved in immune responses to porcine reproductive and respiratory syndrome virus (PRRSV) infection. Anim Genet 2012;43:328-32.

20. Lunney JK, Chen H. Genetic control of host resistance to porcine reproductive and respiratory syndrome virus (PRRSV) infection. Virus Res 2010;154:161-9.

21. Suryawan A, Davis TA. The abundance and activation of mTORC1 regulators in skeletal muscle of neonatal pigs are modulated by insulin, amino acids, and age. J Appl Physiol 2010;109:1448-54.

22. Li M, Tian S, Yeung CKL, et al. Whole-genome sequencing of Berkshire (European native pig) provides insights into its origin and domestication. Sci Rep 2014;4:4678. 